\title{
Gait Analysis in Adult People with Hip Retrotorsion
}

\author{
DongSik Oh \\ Dept. of Physical Therapy, Division of Health Science, Hanseo University, Korea \\ dongsiko@hanseo.ac.kr
}

\begin{abstract}
The purpose of this study was to conduct gait analysis of the hip joints of adults with retrotorsion when walking down stairs. Methods: Ten students with hip retrotorsion were selected from 230 healthy men and women. Results: The range of motion in the retrotorsion group in the sagittal and frontal planes was not significantly different in either joint ( $p>.05)$, but the affected side at the transverse plane had a significantly lower range of motion than the non-affected side ( $p<.05)$. There was no significant difference in either joint in the normal group in all planes ( $p>.05)$. Conclusion: During stair descent in adults with hip retrotorsion, the affected side showed greater internal rotation and a lower abduction angle than the nonaffected side. We expect these findings to help in preventing musculoskeletal disorders that may occur in daily life due to femoral torsion and to offer valuable information regarding walking.
\end{abstract}

Keywords: Descending stairs, Femur, Kinematics, Range of motion, Torsion

\section{Introduction}

Stair walking is a common motion in everyday life and requires more physical load and range of motion in the lower limb joints than walking on flat ground, resulting in higher energy consumption than ordinary walking. Stair descent has also been reported to have a higher fall risk than stair ascent[1][2][3].

The transverse axis of the femoral neck and femoral condyle is tilted forward from the frontal plane by more than 30 degrees at birth, and the angle gradually decreases inwardly until 6 years of age to around 15 degrees in adults, on average. Cases in which the angle of inclination is higher than the normal 15 degrees are called hip antetorsion, and cases in which the angle is reduced to below 8 degrees and tilted backward against the femoral shaft are called hip retrotorsion [4].

Gait patterns resulting from excessive antetorsion or retrotorsion of the femur can lead to gait disorders or early degenerative arthritis, including hip osteoarthritis, hip labral tearing, and patellofemoral pain [5][6][7], and have been reported to affect not only knee stability, but also the waist [8].

Until now, examination of the femoral torsion for hip rotation has been mainly conducted using radiotherapy, but this static evaluation method does not fully reflect the dynamic elements of gait; hence, 3D analysis is now recommended [9][10]. The walking of normal people is symmetrical, but subtle kinematic or kinetic asymmetry can occur [11]. Studying the functional asymmetry of gait may help to explain gait disorder in neurologically impaired patients or in normal people at risk of falling [12], and gait analysis to assess normal gait function may be critical in clinical or rehabilitation processes[13].

Thus, even though many studies of femoral torsion and kinetic studies of stair walking have been conducted, kinematic walking research into hip retrotorsion is insufficient. This study 
therefore aims to analyze the kinematic factors of hip joints during stair descent in young adults with hip retrotorsion, to help prevent future disease and to gather basic data on walking.

\section{Research methods}

\subsection{Subjects}

A total of 230 healthy men and women attending $\mathrm{H}$ University in Chungcheongnam-do were surveyed, and cross-sectional research was conducted on 10 students with hip retrotorsion. A total of 30 men and women were randomly sampled as the normal group, excluding those who had hip antetorsion or hip retrotorsion. The subjects of the study were told the purpose, process, and evaluation methods of this study and voluntarily agreed to participate.

The exclusion criteria were experience of surgery in the lower extremities; difficulty in walking due to pain; congenital malformations; visual, auditory, or neurological disorders; a body mass index (BMI) of 23 or higher in the lower body; a $0.5 \mathrm{~cm}$ or greater difference in the lengths of the legs; and scoliosis, knee varus, or valgus.

\subsection{Measuring tools and equipment}

a. Hip-joint range of motion: The range of motion of the hip joint was measured using a Level Angle Finder (Kraft, USA).

b. Gait analysis: The rotational motion of the hip joint was measured using a Noraxon MyoMotion (Scottsdale, AZ, USA) three-dimensional motion analyzer.

\subsection{Experimental methods}

All subjects flexed their knees 90 degrees, with the pelvis fixed in the supine position, and their hip-joint range of motion was measured using Craig's Test [4]. After the test, only the subjects with hip retrotorsion were selected, and they performed a stair descent. All subjects were instructed to wear comfortable trousers and sneakers, but not slippers. After the sensor was attached while subjects wore the clothes and shoes they had selected for the experiment, they were instructed to use their usual, natural stair-walking motion and were provided an adaptation period of one descent and ascent of 10 stairs. The pelvic sensor was attached above the sacrum, and the femoral sensor was attached to a site above the patella and a quarter below the quadriceps, where the sensor does not change in position due to the contraction of muscles during walking. The subjects were analyzed using a 3D motion analyzer for the motion pattern of the hip joint at the early stage of the stance phase, when the right foot touches the ground, and during stair descent from the first step to the last step. The height and width of each step were $8 \mathrm{~cm}$ and $190 \mathrm{~cm}$, respectively; the total length of the stairs was $300 \mathrm{~cm}$, and the slope was 35 degrees.

\subsection{Analysis method}

All data was analyzed using SPSS version 12.0 (SPSS Inc., Chicago, IL, USA). The Wilcoxon code rank test was used to compare the left and right hip joints of the affected and non-affected sides in both the hip retrotorsion and normal groups. The statistical significance level was set to $\alpha=0.05$.

\section{Results}


3.1. Comparison of the hip-joint range of motion between the affected and non-affected sides in the sagittal, frontal, and transverse planes in the hip retrotorsion group

The joint ranges of motion in the sagittal and frontal planes showed no significant differences between the two sides ( $p>.05)$.

The joint ranges of motion in the transverse plane showed a significant difference, as the range of motion of the affected side decreased more than in the non-affected side $(\mathrm{p}<.05)$ [Table 2].

\subsection{Comparison of the maximum hip-joint angles in the sagittal, frontal, and transverse planes between the affected and non-affected sides in the hip retrotorsion group}

The maximum angles in the sagittal and transverse planes showed no significant differences between the two sides ( $p>.05$ ).

The maximum angle in the frontal plane showed a significant difference, as the abduction on the affected side increased more than on the non-affected side $(\mathrm{p}<.05)$ [Table 3].

\subsection{Comparison of the minimum hip-joint angles in the sagittal, frontal, and transverse} planes between the affected and non-affected sides in the hip retrotorsion group

The minimum angle in the sagittal plane showed no significant difference between the two sides $(\mathrm{p}>.05)$.

The minimum angle in the frontal plane showed a significant difference, as the adduction on the affected side decreased more than on the non-affected side $(\mathrm{p}<.05)$.

The minimum angle in the transverse plane showed a significant difference, as the internal rotation on the affected side decreased more than on the non-affected side $(\mathrm{p}<.05)$ [Table 4].

3.4. Comparison between the left and right sides of the hip joint in the sagittal, frontal, and transverse planes in the normal group

The hip-joint ranges of motion, maximum angles, and minimum angles in the sagittal, frontal, and transverse planes showed no significant differences between the two sides ( $p>.05)$ [Tables $2,3,4]$.

Table 1. The general characteristics of subjects

\begin{tabular}{|c|c|c|}
\hline Variables & Normal $(\mathrm{n}=30)$ & Retrotorsion $(\mathrm{n}=10)$ \\
\hline Age (year) & $23.93 \pm 1.51 \mathrm{a}$ & $22.60 \pm 1.52$ \\
\hline Sex(Male/Female) & $12 / 18$ & $7 / 3$ \\
\hline Side(left/right) & $0 / 30$ & $6 / 4$ \\
\hline BMI(kg/m2) & $20.83 \pm 0.76$ & $20.95 \pm 1.02$ \\
\hline Leg length discrepancy & $83.91 \pm 3.47$ & $83.7 \pm 4.05$ \\
\hline External Hip Rotation $^{\circ}{ }^{\circ}$ & $43.13 \pm 5.71$ & $64.25 \pm 5.37$ \\
\hline Internal Hip Rotation $\left.^{\circ}{ }^{\circ}\right)$ & $32.52 \pm 6.45$ & $16.75 \pm 2.06$ \\
\hline
\end{tabular}

${ }^{a}$ Mean \pm Standard deviation. BMI: Body Mass Index

Table 2. Comparison of range of motion of hip in the sagittal, frontal, transverse planes

\begin{tabular}{|l|l|l|l|}
\hline \multirow{2}{*}{} & \multirow{2}{|c|}{ Range of motion } & $\mathrm{p}$ \\
& & Affect/Lt & Non-affect/Rt
\end{tabular}




\begin{tabular}{|c|c|c|c|c|}
\hline \multirow{2}{*}{ Sagittal Plane (Fl/Ex) } & Retrotorsion & $24.22 \pm 5.06^{\mathrm{a}}$ & $25.49 \pm 2.24$ & 0.11 \\
\cline { 2 - 5 } & Normal & $27.43 \pm 4.59$ & $26.44 \pm 4.08$ & 0.14 \\
\hline \multirow{2}{*}{ Frontal Plane (Ab/Ad) } & Retrotorsion & $15.87 \pm 6.48$ & $15.91 \pm 6.19$ & 0.72 \\
\cline { 2 - 5 } & Normal & $7.58 \pm 2.73$ & $7.77 \pm 2.52$ & 0.62 \\
\hline \multirow{2}{*}{ Transverse Plane (In R/Ex R) } & Retrotorsion & $11.55 \pm 7.77$ & $15.38 \pm 8.25$ & $0.02^{*}$ \\
\cline { 2 - 5 } & Normal & $16.34 \pm 4.61$ & $16.67 \pm 3.17$ & 0.26 \\
\hline
\end{tabular}

${ }^{a}$ Mean \pm Standard deviation.

Fl: Flexion, Ex: Extension, Ab: Abduction, Ad: Abduction, In R: Internal Rotation, Ex R: External Rotation ${ }^{*} p<.05$

Table 3. Comparison of maximum angle of hip in the sagittal, frontal, transverse planes

\begin{tabular}{|c|c|c|c|c|}
\hline \multirow{2}{*}{ Sagittal Plane (Fl/Ex) } & & \multicolumn{2}{|c|}{ Maximum Angle } & \multirow{2}{*}{$\mathrm{p}$} \\
\cline { 3 - 5 } & & Affect/Lt & Non-affect/Rt & 0.81 \\
\cline { 3 - 5 } & Retrotorsion & $36.02 \pm 4.40^{\mathrm{a}}$ & $36.40 \pm 4.34$ & 0.08 \\
\hline \multirow{2}{*}{ Frontal Plane (Ab/Ad) } & Normal & $34.94 \pm 9.57$ & $36.20 \pm 8.39$ & $0.04^{*}$ \\
\cline { 2 - 5 } & Retrotorsion & $11.02 \pm 6.88$ & $8.27 \pm 6.05$ & 0.48 \\
\hline \multirow{2}{*}{ Transverse Plane (In R/Ex R) } & Normal & $1.18 \pm 3.47$ & $1.94 \pm 3.27$ & 0.07 \\
\cline { 2 - 5 } & Retrotorsion & $15.11 \pm 7.04$ & $13.84 \pm 4.82$ & 0.62 \\
\hline
\end{tabular}

${ }^{a}$ Mean \pm Standard deviation.

Fl: Flexion, Ex: Extension, Ab: Abduction, Ad: Abduction, In R: Internal Rotation, Ex R: External Rotation ${ }^{*} p<.05$

Table 4. Comparison of minimum angle of hip in the sagittal, frontal, transverse planes

\begin{tabular}{|c|c|c|c|c|}
\hline \multirow{2}{*}{ Sagittal Plane (Fl/Ex) } & & \multicolumn{2}{|c|}{ Minimum Angle } & \multirow{2}{*}{$\mathrm{p}$} \\
\cline { 3 - 5 } & Retrotorsion & $11.80 \pm 4.76^{\mathrm{a}}$ & $10.91 \pm 3.88$ & 0.24 \\
\cline { 2 - 5 } & Normal & $7.51 \pm 9.62$ & $9.76 \pm 7.94$ & 0.07 \\
\hline \multirow{2}{*}{ Frontal Plane (Ab/Ad) } & Retrotorsion & $-4.85 \pm 2.10$ & $-7.64 \pm 2.06$ & $0.01^{*}$ \\
\cline { 2 - 5 } & Normal & $-6.40 \pm 4.06$ & $-5.83 \pm 3.09$ & 0.48 \\
\hline \multirow{2}{*}{ Transverse Plane (In R/Ex R) } & Retrotorsion & $3.56 \pm 8.41$ & $-1.54 \pm 7.30$ & $0.00^{*}$ \\
\cline { 2 - 5 } & Normal & $-3.11 \pm 5.16$ & $-3.63 \pm 5.92$ & 0.55 \\
\hline
\end{tabular}

${ }^{a}$ Mean \pm Standard deviation.

Fl: Flexion, Ex: Extension, Ab: Abduction, Ad: Abduction, In R: Internal Rotation, Ex R: External Rotation ${ }^{*} p<.05$ 


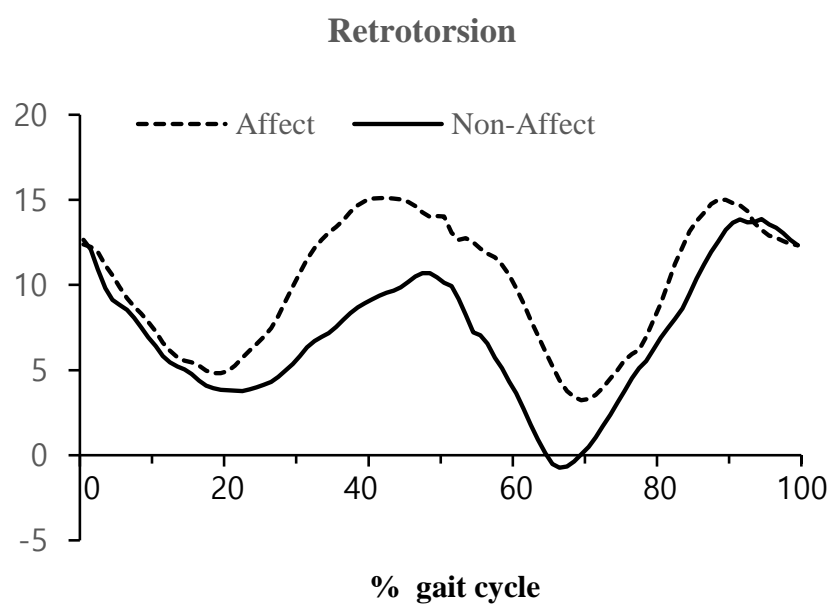

Figure 1. Gait cycle on transeverse plane of normal group

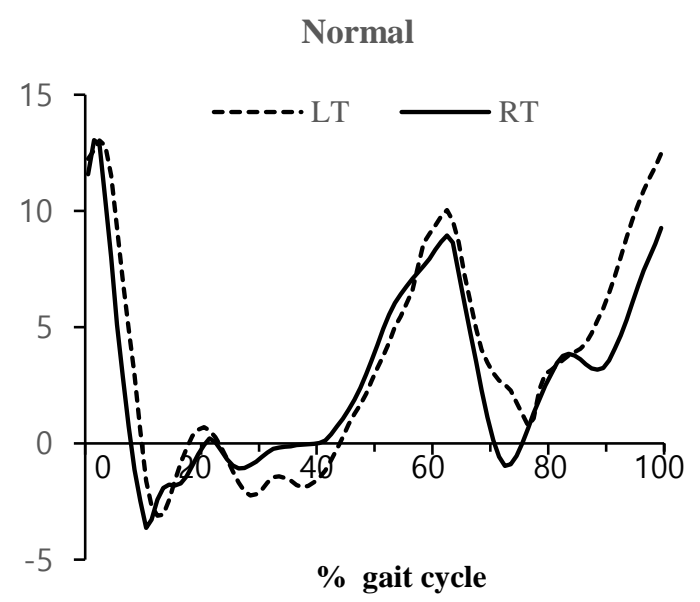

Figure 2. Gait cycle on transeverse plane of retrotosion group

\section{Discussion}

This study investigated the motion pattern of the hip joint during stair descent in young adults with hip retrotorsion. Kwon et al.[14] reported that hip motion on flat ground occurs mainly in the sagittal plane, and that the greatest flexion occurs at the beginning of the stance phase and at complete extension during the rest of the stance phase. Furthermore, in the frontal and transverse planes, the width of motion is small and the femur and pelvis rotate simultaneously. However, according to Kim et al. [15], unlike walking on flat ground, the hip joint is more flexed on both the left and right sides than in the sagittal plane during the entire stance phase. Protopapadaki et al. [16] reported that the hip and knee joints flex in the sagittal plane during the entire stance phase during stair descent in young adults, which is consistent with the results of this study. The hip-joint maximum and minimum angles in the sagittal plane did not differ between the affected and non-affected sides of the hip retrotorsion group nor between the left and right sides of the normal group. However, the minimum and maximum angles of the hip 
joints were flexed during the stair descents of both the hip retrotorsion group and the normal group, suggesting that they flexed the hip joints rather than extending them during their gait.

According to the results of this study, the maximum hip-joint angle in the frontal plane of the hip retrotorsion group increased abduction more on the affected side than on the nonaffected side, but the minimum hip-joint angle decreased adduction more on the affected side than on the non-affected side. However, there was no difference in the angle between the left and right sides in the frontal plane of the normal group. Bruderer-Hofstetter et al. [9] reported that hip adduction moment increased during walking on flat ground in a hip antetorsion group but that further research would be required to determine whether adduction is increased by hip antetorsion or whether abduction is increased by hip retrotorsion.

In the transverse plane, there is a slight anatomical external rotation of the hip joint, but internal rotation occurs during the stair descent motion, and most of the internal rotations occur during the stance phase [14][15]. However, the hip retrotorsion group showed a difference from the normal group in this study, with the internal rotation and range of motion of the affected side decreasing more than for the non-affected side in the transverse plane. The normal and hip retrotorsion groups showed clearly different walking patterns during the entire gait cycle. In the normal group, the internal rotation increased in the left and right sides during the initial stance phase, but in the hip retrotorsion group, external, rather than internal, rotation occurred. These results are consistent with previous studies that found that external rotation increased due to the restriction of internal rotation on the affected side of the hip retrotorsion group compared to the normal group [17][18]. However, during the swing phase, the normal and hip retrotorsion groups showed similar walking patterns on both the affected and non-affected sides, and the hip retrotorsion group walked with more internal rotation of the non-affected side than the affected side.

As discussed above, the normal and hip retrotorsion groups showed different walking patterns; the rotation pattern of the hip joint should therefore be examined and evaluated carefully, because it can seriously damage the function of the musculoskeletal system by causing hip and knee deviations as well as waist deviations.

The limitations of this study are as follows:

It is difficult to generalize the results of this study because of the small number of subjects with hip retrotorsion and because only the impact of hip retrotorsion on the transverse plane of the hip joint was emphasized. Results may also vary depending on the data collection methods and differences in the subjects, staircases, and motion analysis devices. Furthermore, there may be differences in the strides and walking methods of Asians and Westerners [19][20][21]. Therefore, to compensate for these limitations, follow-up studies should conduct kinematic and kinetic analyses of various joints for subjects with hip antetorsion as well as those with hip retrotorsion.

\section{Conclusion}

Young adults with hip retrotorsion showed more flexion than extension of the hip joint in the sagittal plane during stair descent. In the frontal and transverse planes, the affected side of the hip retrotorsion group showed lower internal rotation than those on the non-affected side. The findings of this study are expected to be used as basic data for the prevention of musculoskeletal diseases that can be caused by femoral torsion in daily life as well as for research on walking. 


\section{References}

[1] H. J. Bennett and S. Zhang, G. Shen, J. T. Weinhandl, M. R. Paquette, J. Reinbolt, D. P. Coe. "Effects of toein and wider step width in stair ascent with different knee alignments," Med Sci Sports Exerc, vol.49, no.3, (2017)

[2] J. K. Startzell and D. A. Owens, L. M. Mulfinger, P. R. Cavanagh, "Stair negotiation in older people: A review," Journal of the American Geriatrics Society, J Am Geriatr Soc, vol.48, no.5, (2000) DOI: 10.1111/j.15325415.2000.tb05006.x

[3] K. Singhal, J. Kim, J. Casebolt, S. Lee, K. H. Han, and Y. H. Kwon, "Kinetic comparison of older men and women during walk-to-stair descent transition," Gait posture, vol.40, no.4, (2014) DOI: 10.1016/j.gaitpost.2014.07.004

[4] R. B. Souza and C. M. Powers, "Concurrent criterion-related validity and reliability of a clinical test to measure femoral anteversion,” J Orthop Sports Phys Ther, vol.39, no.8, (2009) DOI: 10.2519/jospt.2009.2996

[5] D. Tönnis and A. Heinecke, "Current concepts review - acetabular and femoral anteversion: relationship with osteoarthritis of the hip,” J. Bone Joint Surg Am, vol.81, no.12, pp.1747-1817, (1999)

[6] M. C. Worlicek, M. L. Wörner, B. S. Craiovan, F. Zeman, J. Grifka, T. F. Renkawitz, and M. Weber, "Lesser trochanter size on plain anteroposterior radiographs correlates with native femoral anteversion," J Arthroplasty, vol.32, no.9, pp.2892-2897, (2017) DOI: 10.1016/j.arth.2017.03.054

[7] T. Bell-Jenje, B. Olivier, W. Wood, S. Rogers, A. Green, and W. McKinon. "The association between loss of ankle dorsiflexion range of movement, and hip adduction and internal rotation during a step down test," Man Ther, vol.21, pp.256-261, (2016) DOI: 10.1016/j.math.2015.09.010

[8] M. T. Cibulka, M. J. Strube, D. Meier, M. Selsor, C. Wheatley, N. G. Wilson, and J. J. Irrgang, "Symmetrical and asymmetrical hip rotation and its relationship to hip rotator muscle strength," Clin Biomech, vol.25, no.1, pp.56-62, (2010) DOI: 10.1016/j.clinbiomech.2009.09.006

[9] C. Radler, A. Kranzl, H. M Manner, M, Höglinger, R, Ganger, and F. Grill, “Torsional profile versus gait analysis: Consistency between the anatomic torsion and the resulting gait pattern in patients with rotational malalignment of the lower extremity," Gait Posture, vol.32, no.3, pp.405-410, (2010) DOI: 10.1016/ j.gaitpost.2010.06.019

[10] M. Bruderer-Hofstetter, V. Fenner, E. Payne, K. Zdenek, H. Klima, and R. Wegener, "Gait deviations and compensations in pediatric patients with increased femoral torsion,” J Orthop Res, vol.33, no.2, pp.155-162, (2015) DOI: 10.1002/jor.22746

[11] K. A. Shorter, J. D. Polk, K. S. Rosengren, and E. T. Hsiao-Wecksler, "A new approach to detecting asymmetries in gait," Clin Biomech, vol.23, no.4, pp.459-467, (2008) DOI: 10.1016/j.clinbiomech.2007.11.009

[12] [J. D. Polk, R. M. Stumpf, and K. S. Rosengren, “Limb dominance, foot orientation and functional asymmetry during walking gait,” Gait Posture, vol.52, pp.140-146, (2017) DOI: 10.1016/j.gaitpost.2016.11.028

[13] P. R. Worsley, G. Whatling, D. Barrett, C. Holt, M. Stokes, and M. Taylor, "Assessing changes in subjective and objective function from pre- to post-knee arthroplasty using the cardiff dempster-shafer theory classifier," Comput Methods Biomech Biomed Engin, vol.19, no.4, pp.418-427, (2016) DOI: 10.1080/ 10255842.2015.1034115

[14] O. Y. Kwon, J. H. Won, J. S. Oh, L. W. Lee, and S. J. Kim, "Effects of the hip internal rotation gait on gluteal and erector spinae muscle activity," Phys Ther Korea, vol.13, no.1, pp.24-31, (2006)

[15] Y.S. Kim, E. J. Kim, and C. J. Seo, “The comparative analysis of EMG and gait patterns of lower extremities during going up stairs and down," Kor J Phys Edu., vol.45, (2006)

[16] A. Protopapadaki, W. I. Drechsler, M. C. Cramp, F. J. Coutts, and O. M. Scott, "Hip, knee, ankle kinematics and kinetics during stair ascent and descent in healthy young individuals," Clin Biomech, vol.2, no.22, pp.203210, (2007) DOI: 10.1016/j.clinbiomech.2006.09.010 
[17] A. Uding, N. J Bloom, P. K Commean, T. J. Hillen, J. D. Patterson, J. C. Clohisy, and M. Harris-Hayes, "Clinical tests to determine femoral version category in people with chronic hip joint pain and asymptomatic controls,” Musculoskelet Sci Pract., vol.39, pp.115-122, (2019) DOI: 10.1016/j.msksp.2018.12.003

[18] F. Accadbled and J. Cahuzac, "In-toeing and out-toeing," Rev Prat, vol.56, no.2, (2006)

[19] D. Samuel, P. Rowe, V. Hood, and A. Nicol, "The biomechanical functional demand placed on knee and hip muscles of older adults during stair ascent and descent," Gait posture, vol.34, no.2, pp.239-244, (2011) DOI: 10.1016/j.gaitpost.2011.05.005

[20] O. S. Mian, J. M. Thom, M. V. Narici, and V. Baltzopoulos, "Kinematics of stair descent in young and older adults and the impact of exercise training," Gait Posture, vol.25, no.1, pp.9-17, (2007) DOI: 10.1016/j.gaitpost.2005.12.014

[21] T. Ryu, H. S. Choi, H. Choi, and M. K. Chung, "A comparison of gait characteristics between Korean and Western people for establishing Korean gait reference data,” Int J Ind Ergon, vol.36, no.12, pp.1023-1030, (2006) DOI: 10.1016/j.ergon.2006.09.013 